La artesanía en Andalucía 03

\title{
El cristal de Sevilla. La fábrica de vidrios de La Trinidad
}

Macarena Hernández Ramírez. Profesora del Dpto. de Ciencias Sociales. Universidad Pablo de Olavide 


\section{La relación entre artesanía y patrimonio, entre usos y cultura, es algo fundamentalmente dinámico}

Hablar de la fábrica de vidrio de La Trinidad en Sevilla es mucho más que relatar el proceso de elaboración de una sustancia obtenida por fusión y rápido enfriamiento de sílices, calcio, sodio-potasio y diversos tipos de óxidos. Hablar de La Trinidad es hablar de la historia de una manufactura y sus protagonistas; es relatar la trayectoria de una artesanía; de ahí que desde hace bastante tiempo, y desde muy diferentes sectores, se venga afirmando que la historia de los hombres y de las pocas mujeres que han fabricado el vidrio en La Trinidad (en el último siglo) ayuda a recomponer la historia de la ciudad.

A pesar de ello y de contar con el reconocimiento oficial (vía inscripción en el Catálogo General del Patrimonio Histórico Andaluz desde septiembre de 2001) para ser parte de la historia y del patrimonio cultural de la ciudad de Sevilla, lo cierto es que La Trinidad, su pasado, su presente y, por ende, su futuro, sigue siendo desconocida para gran parte de los teóricos depositarios de ese patrimonio, es decir, para los andaluces.

\section{Artesanos del vidrio soplado en La Trinidad}

La fabricación de vidrio soplado se conoce aproximadamente desde el siglo I a.C., cuando se descubre un procedimiento revolucionario: soplar el vidrio y expandirlo hasta lograr todo tipo de formas. El elemento fundamental consiste en un tubo o caña de hierro con una boquilla en un extremo. El soplador de vidrio toma una pequeña porción con el extremo de la caña y le da forma más o menos cilíndrica haciéndola girar sobre una plancha de hierro colado. Posteriormente comienza a soplar a través de la caña para formar una burbuja con la masa vítrea y obtener a partir de ella la forma y el espesor deseado, moldeando y recalentan-

- Horno. Fábrica de vidrios de Guadarte (Alcalá de Guadaira, Sevilla) / Germán Matas do la masa junto al horno. Éste ha sido el método más extendido hasta bien entrado el siglo XIX.

En la actualidad se mantienen dos formas de fabricación: a) vidrio industrial y b) vidrio artesanal, sin mecanización, o en forma de manufacturas que han conservado técnicas tradicionales o introducido nuevas, pero siempre de manera artesanal, donde la actividad del agente que lo fabrica, del vidriero, preside el proceso.

La fábrica de La Trinidad estaría en el intermedio entre ambas opciones señaladas para la producción de vidrio, es decir, hablamos de una producción semiindustrial,mucho más próxima en sus técnicas y usos productivos a la artesanía que a la industria en cadena.

La Trinidad comparte características con el resto de industrias instaladas en la ciudad a comienzos de siglo, y se sitúa dentro de uno de los espacios industriales que caracterizan la Sevilla de principios del XX: la avenida de Miraflores -entonces terreno de huertas, arroyos como el Tagarete y lagunas como la de los Patos o la del Arzobispo- que para este entonces nace como uno de los ejes urbanos resultantes de la expansión de la ciudad hacia el norte y el oeste.

La historia social de la fábrica comienza con la misma persona de su propulsor, el ingeniero y militar Luis Rodríguez Caso. El 15 de Octubre de 1900 Rodríguez Caso presenta en el Ayuntamiento de Sevilla la solicitud para instalar una fábrica de vidrios y cristales, huecos y planos.

Con la contratación del primer director técnico (Francisco Thirión) comienza parte de la historia humana de la fábrica: con él llega el conocimiento del oficio, y además se inicia: a) por un lado, una de las sagas de vidrieros más relevantes en la historia de La Trinidad -ya que sus hijos han sido miembros de la fábrica hasta el año 1971, año en que se 
jubiló el más pequeño- y b) el contacto con toda la tradición vidriera del levante español, hecho que sin duda favorecería la llegada de obreros y de técnicas de otros centros productores.

La producción comenzó en 1902, desde ese momento la historia de la fábrica y de la actividad ha sido la historia de un grupo de vecinos, inscritos en el contexto social, económico y cultural que marcaba la ciudad y el momento histórico que se vivía.

En 1920 la fábrica cambia de dueño y pasa a Fernando Barón (Conde de Colombí). Son tiempos de cambios económicos, que sin duda afectan a La Trinidad y a su fisonomía. En 1928 La Trinidad vuelve a cambiar de dueño, pasando a manos de la familia Medina Benjumea.

En 1930 la fábrica llega a tener 500 empleados, que en ningún momento están ajenos al estado de cambios sociales y políticos de entorno. Es una de las décadas principales en cuanto a transformacio nes sociales de la fábrica, marcada por una falta de acuerdo entre la empresa y los trabajadores que imposibilita una producción regular, y que provoca que Medina Benjumea ceda la fábrica a los trabajadores por un período de diez años. Éste fue el punto de arranque de la Sociedad Cooperativa Obrera La Trinidad, con fecha de constitución en el 16 de diciembre de 1934. Este hecho supone otro de los rasgos distintivos de la historia humana y obrera de esta fábrica, pues este sistema de funcionamiento se ha mantenido en La Trinidad hasta el año 1993 cuando se constituye como Sociedad Anónima Laboral, perviviendo incluso durante la guerra civil y los duros años de la posguerra.

Una vez que ha terminado la guerra civil, hacia la década de los cuarenta, se relanza la actividad de la fábrica, y en 1945 la Unión Nacional de Cooperativas Industriales compra el patrimonio de la fábrica a la familia Medina Benjumea. Un año después, en 1946, la Cooperativa adquiere el patrimonio a la Unión Nacional de Cooperativas Obreras, pagando el primero de una larga lista de créditos hipotecarios.

En el intervalo entre las décadas de los años 50 a los 70, La Trinidad asiste a un periodo de cierta tranquilidad en su producción, al unísono con el mercado nacional y la economía de los clientes más cercanos a la fábrica. Una proyección que no fue duradera, pues la llegada de nuevos productos al mercado y técnicas de fabricación, unido a las consecuencias de la crisis de la economía española de 1973, desembocan en una imparable decadencia de La Trinidad hasta el fin de su actividad -como tal fábrica- en el año 1999. Durante todo este período, La Trinidad ha mantenido el mismo modo productivo: una fabricación, semindustrial, más cercana al concepto de artesanía que a la industria, que poco ha variado a través del tiempo.

Al realizar una secuencia que nos permita considerar la dimensión cultural de la producción de esta artesanía en La Trinidad, necesariamente hemos de contemplar todas y cada una de las especificidades que ha presentado la producción de vidrio en esta fábrica. Aunque aquí no entraremos en la descripción precisa de los momentos y pasos de la producción -desde las mezclas al decorado-, ni en la descripción pormenorizada de cada una de las herramientas empleadas ${ }^{1}$, u oficios de los hombres y mujeres que daban sentido a la producción, sí destacamos su papel esencial dentro de la consideración patrimonial. Sin duda alguna, la especificidad de la producción de vidrio fuerza la existencia de instrumentos (herramientas) y conocimientos (oficios) exclusivos del tipo de vidrio, razón por la que en La Trinidad han convivido y se han empleado técnicas y herramientas concebidas "in situ" para crearlo.

Es esta necesidad de precisión, de ajustarse a lo específico-además de por el ahorro económico que suponía-, la que justifica la existencia, desde su instalación, de diversos talleres relacionados directamente con la fabricación de materiales y herramientas específicos empleados en la producción de vidrio. Son talleres que se han mantenido hasta el cese de la actividad: taller de albañilería, taller de carpintería, y de manera destacada recordamos el taller de herrería, donde los herreros elaboraban parte esencial del patrimonio de la fábrica: los más de tres mil moldes inventariados en La Trinidad²

En cada uno de estos talleres se encontraban, además de las herramientas diseñadas para la fabricación vidriera, cada uno de los actores desarrollando y dando contenido a la diferencia del oficio ${ }^{3}$.

Ya fuera por la cantidad de horas que ocupaban los obreros dentro de la fábrica -con diferentes ciclos y horarios, según la trayectoria de la misma-, o por la homogeneidad en la procedencia y aspiraciones sociales, los obreros constituían un grupo que tenía el espacio de la fábrica como lugar para la expresión de una buena parte de su sociabilidad. Pensemos en contextos y coyunturas cotidianas compartidas por estos hombres y mujeres, que los señalaban como grupo social, portador de una línea de transmisión de conocimientos (científicos, tecnológicos, artesanales) sin los cuales no se hubiera podido mantener la actividad.

Estos oficios, el uso que de estas herramientas han mantenido estos vidrieros, unido a las propiedades del cristal con el que trabajaban, es lo que ha otorgado especificidad al vidrio soplado en la fábrica, y lo que justificaba, principalmente, la decisión de la Administración a la hora de considerar Lugar de Interés Etnológico (BOJA 110 de 22 de septiembre de 2001) a la fábrica de vidrio de la Trinidad. Desde entonces La Trinidad es, oficialmente, patrimonio de Andalucía.

\section{Vigencias en la historia de La Trinidad y del patrimonio de la ciudad}

Tal y como la ley dispone, la declaración de La Trinidad venía acompañada de unas acertadas instrucciones particulares con las que actuar ante 


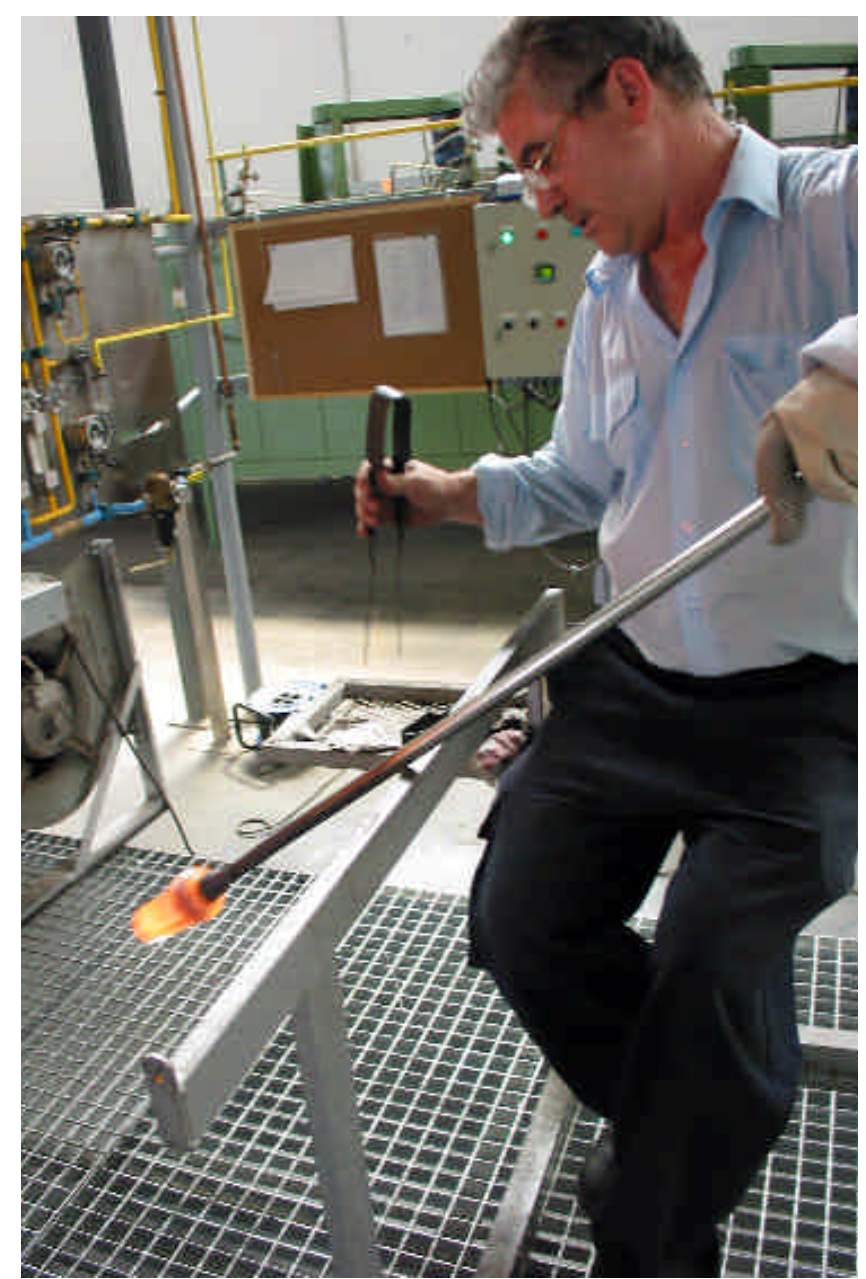

๑ Vidriero P. Torralbo realizando una pieza. Guadarte (Alcalá de Guadaira, Sevilla) / Germán Matas

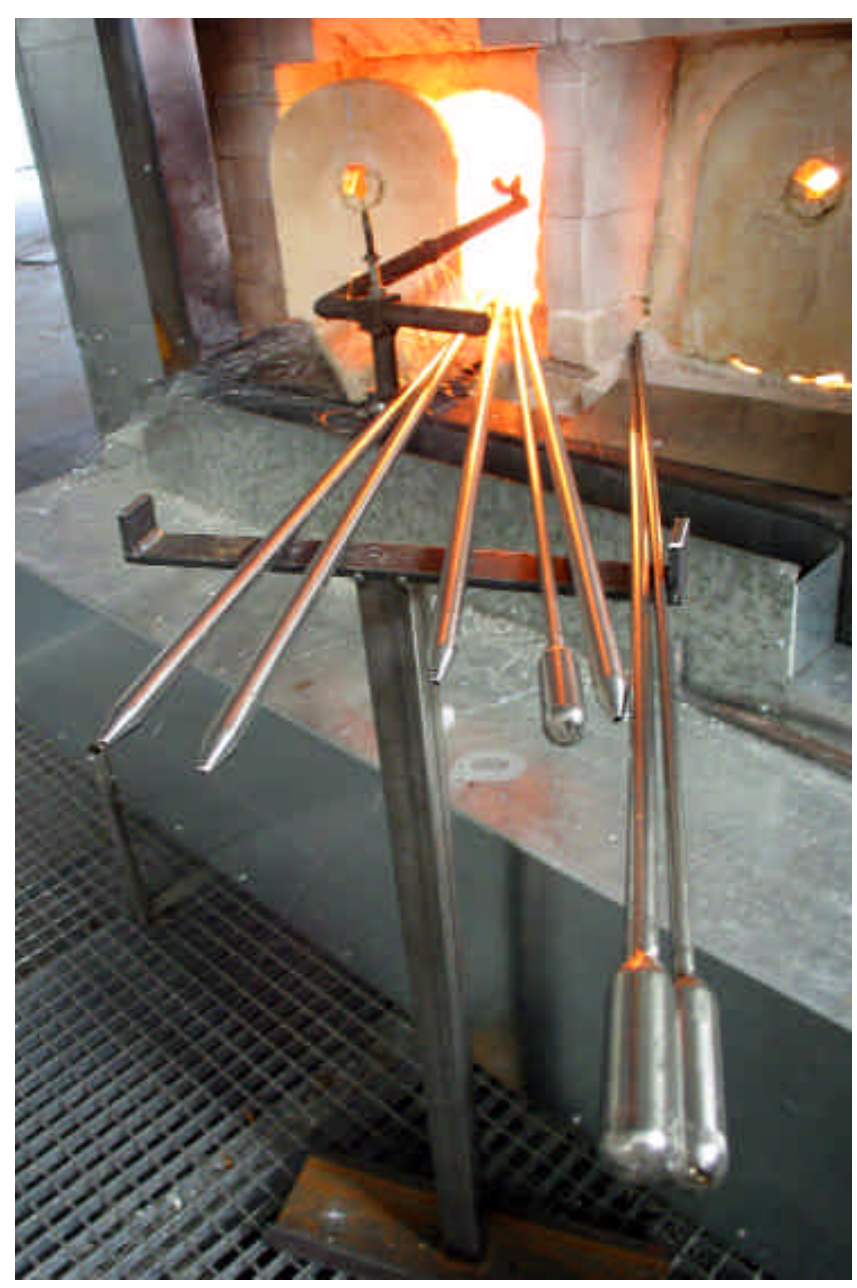

ه Cañas calentándose / Germán Matas

Los obreros constituían un

grupo que tenía el espacio de la fábrica como lugar para la

expresión de una buena parte de su sociabilidad 


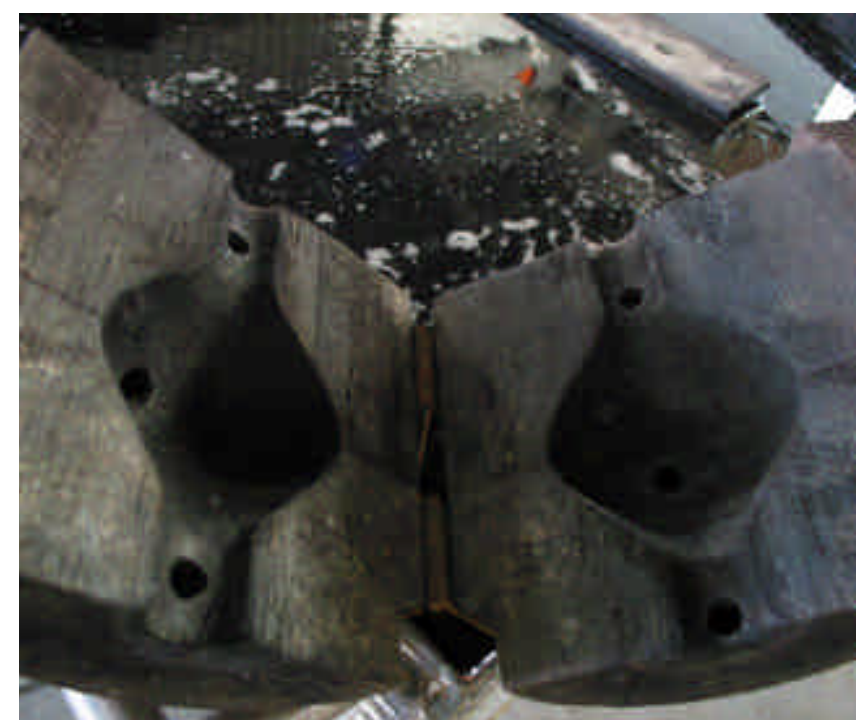

(1) Molde de madera / Germán Matas
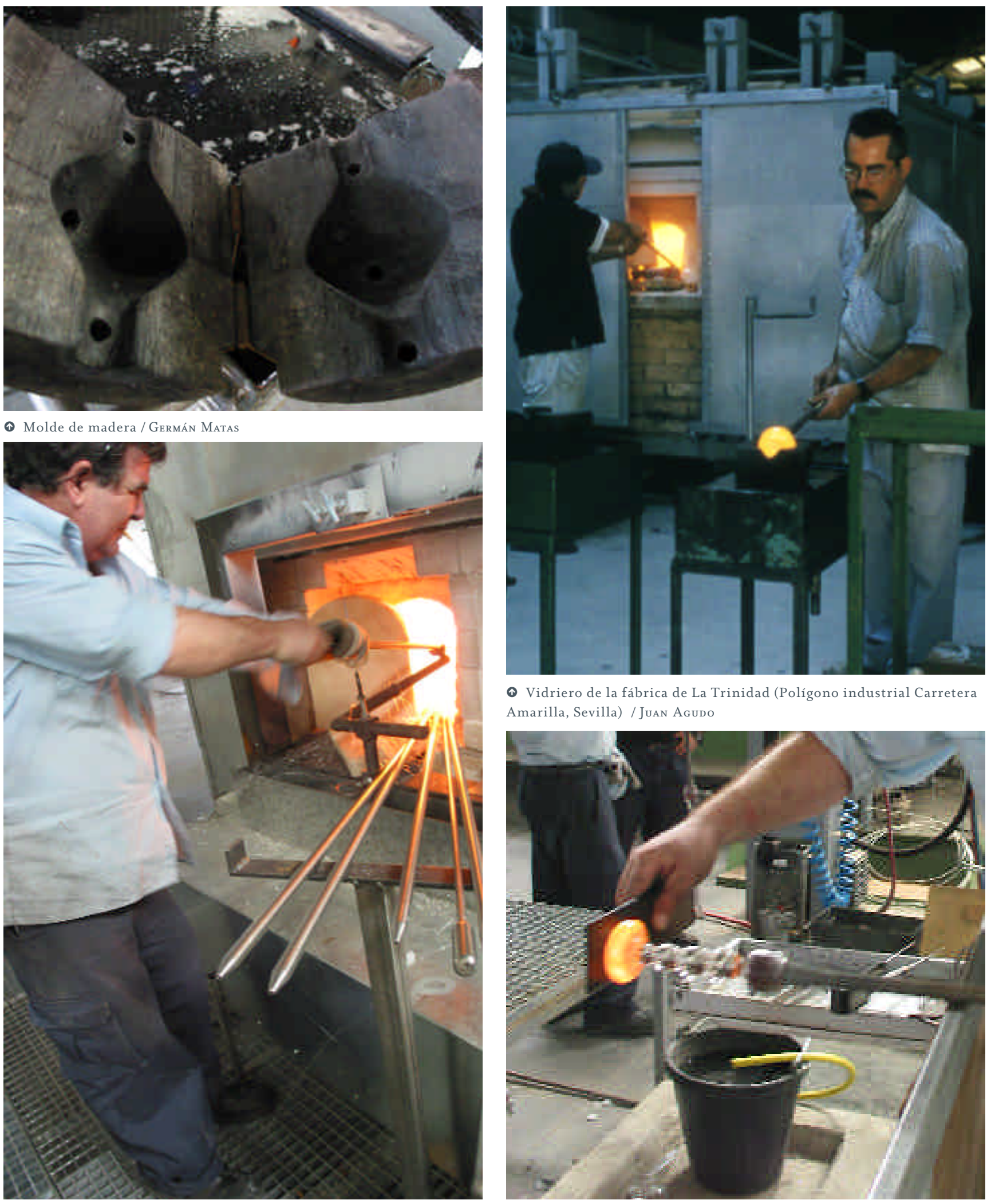

(1) Vidriero de la fábrica de La Trinidad (Polígono industrial Carretera Amarilla, Sevilla) / Juan Agudo

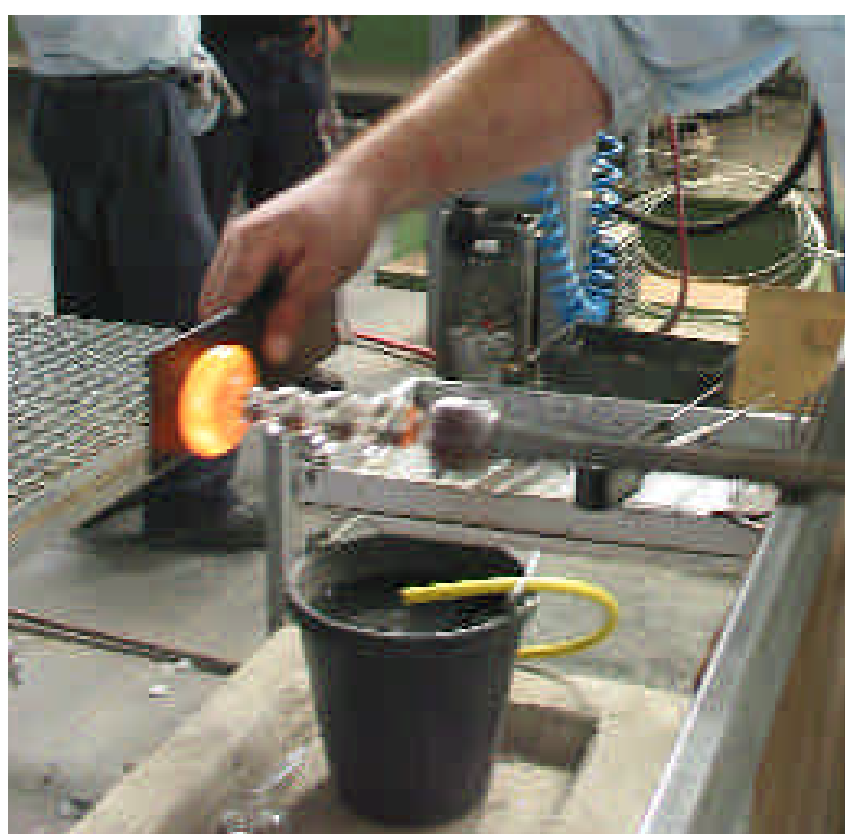

(1) Proceso de elaboración de una pieza / Germán Matas

(『) Realización de una pieza. Detalle / Germán Matas 
lo que la realidad manda. Una realidad que nos sitúa en el momento de la incoación del expediente (septiembre de 1999), justo cuando cesa la actividad en la fábrica, se abandona el espacio y se divide su historia: Ahora en La Trinidad encontramos cómo, por un lado, se presenta de manera homogénea y estática el contendor de este patrimonio, es decir, el inmueble de la fábrica; un goloso solar, donde a fecha de febrero de 2006 todavía no se ha acometido ninguna intervención visible, y que más bien parece mantenerse a la espera de una importante y anunciada actuación inmobiliaria en concordancia con su categoría patrimonial.

Y por otro, vemos cómo lejos de este espacio histórico y común sobreviven -de manera diversa y compleja- contenidos y agentes de este patrimonio. Las artesanías y los protagonistas de La Trinidad sí parecen haber hallado algún camino posible y actual por el que transitar. Parte de los 67 obreros que permanecían en la fábrica en el momento de la clausura (18 exactamente) decidió 3 años después del cierre aventurarse a reiniciar la actividad en otra sede, en el polígono industrial Carretera Amarilla, y de este modo retomar las herramientas, los conocimientos y la historia para defenderse, como artesanos, en la actualidad del mercado. Ellos, como agentes vivos de ese patrimonio, y haciendo uso de las disposiciones que marca y permite la propia ley de Patrimonio Histórico de Andalucía, solicitaron poder mantener vivo y usar parte de ese patrimonio con el que han trabajo a lo largo de un siglo. Actualmente son los que disfrutan, además del nombre y el logo tradicional de la fábrica, de las más específicas herramientas que se precisan para la producción del vidrio.
Por este motivo, principalmente, mantienen pleitos legales con el otro bloque de compañeros y protagonistas de La Trinidad que decidieron seguir siendo vidrieros. Me refiero a un pequeño grupo de antiguos trabajadores de La Trinidad que buscó poco tiempo después el amparo empresarial y económico dentro una estructura comercial e industrial mayor, más concretamente en el seno de la empresa alcalareña Guadarte. Lo que comenzó siendo la iniciativa personal de uno de ellos, se está convirtiendo en el futuro profesional de los más de veinte vidrieros que componen este grupo. En esta empresa parecen haber encontrado la mejor plataforma para participar -con su producto y sus formasen las lógicas de producción, circulación y consumo de las artesanías ${ }^{4}$.

Es ésta una situación que, a pesar de haber sido presentada de forma tan somera en este breve texto, nos hace pensar, desde hace algún tiempo, en la razón, el discurso, la práctica y sobre todo las vigencias de lo que denominamos y usamos como patrimonio cultural. Una reflexión que nos invita (además de a centrar en este aspecto nuestro principal interés en el análisis del patrimonio) a cuestionarnos si realmente son las historias actuales de La Trinidad actividades compatibles con el mantenimiento de los valores del bien, tal y como ordenan las instrucciones particulares de la declaración. El relato de su trayectoria y el seguimiento de su actualidad nos llevan a responder, más que desde la oficialidad, desde los valores del bien, desde la relación - entendemos representada en la fábrica de La Trinidad - entre artesanía y patrimonio, entre usos y cultura, como algo fundamentalmente dinámico, y a pensar que esta forma de hacer vidrio soplado sigue siendo un patrimonio cultural de nuestra ciudad.

\section{Bibliografía}

ALMUEDO PALMA, J. (1996) Ciudad e Industría. Sevilla 850 1930. Sevilla: Diputación, 1996

FERNÁNDEZ DE PAZ, E. (1999) La documentación y protección de las artesanías como actuaciones sobre el Patrimonio Etnográfico. En AGUILAR CRIADO, E. (ed.) Patrimonio etnológico: nuevas perspectivas de estudia Instituto Andaluz del Patrimonio Histórico, Sevilla, 1999, pp.170-191

\section{Notas}

${ }^{1}$ Aspectos todos ellos perfectamente expuestos y analiza dos en la literatura existente sobre el tema, de entre la que de manera especial señalamos, y en este apartado, la obra del profesor Sobrino (1997).

2 Estos moldes recogen una parte importante de la trayectoria del patrimonio tecnológico, no sólo de la fábrica, sino de la ciudad. A través de estos moldes analizamos los productos que realizaba La Trinidad, sus clientes (las principales bodegas y licoreras de la región y la provincia, así como
GONZÁLEZ CORDÓN, A. (1 985) Vivienda y Ciudad, 1849-1929. Sevilla: Ayuntamiento, Centro Municipal de Documentación Histórica, 1985

GONZÁLEZ PEÑA, Ma. L. (1984) Vidrios Españoles. Madrid: Editora Nacional, 1984

GUILLAUME, J (1958) Las artes del fuego. Barcelona: Vergara Editorial, S.A., 1958
PLANELL, L. (1948) Vidrio: historia, tradición y arte, volumen II. Barcelona, 1948

SOBRINO SIMAL, J. (1997) Patrimonio Tecnológico de Andalucía. Sevilla: Consejería de Educación y Ciencia, 1997 del extranjero, fabricantes de productos medicinales, com pañías aéreas, hermandades) sus transformaciones, y en definitiva, parte de la historia de la Sevilla del siglo XX, tal y como se puede ver al contemplar los diferentes catálogos de ventas que se conservan.

${ }^{3}$ Mezclador de materias primas, fundidores, maestro vidriero o maestro de gran plaza (de quien depende el aprendiz de vidriero, vidrieros tronchadores y control de calidad, o "escogedores"), doradores, talladores, especialista en calco - manía, empaquetadores, encargado de taller de moldería, metalúrgicos y soldadores, personal de administración, dirección y comercialización, son los oficios que completaban la secuencia de producción.

${ }^{\mathbf{4}}$ En este sentido, me gustaría referir esta empresa de manera destacada, y sobre todo su capacidad y habilidad compleja a la hora de "producir" industrialmente manufacturas y artesanías. 